\title{
The Provable Constructive Effect of Diffusion Switching Mechanism in CLEFIA-type Block Ciphers
}

\author{
Qingju Wang ${ }^{\mathrm{a}, \mathrm{b}}$, Andrey Bogdanov ${ }^{\mathrm{b}}$ \\ ${ }^{a}$ Shanghai Jiao Tong University, Department of Computer Science and Engineering, N0.800, Dongchuan Road, \\ Shanghai, 200240, China \\ ${ }^{b}$ Katholieke Universiteit Leuven, ESAT/COSIC and IBBT, Kasteelpark Arenberg 10, 3001 Leuven, Belgium
}

\begin{abstract}
CLEFIA is a popular recent block cipher designed by Sony Corporation, accepted as a lightweight encryption algorithm of the new ISO/IEC 29192-2 standard, and proposed as a Japanese e-Government recommendation cipher CRYPTREC candidate.

Provable security properties of cryptographic design are crucial in any security evaluation. Providing lower bounds on the number of active S-boxes in differential and linear characteristics has been one of the few important provable properties that can be formally shown for block ciphers and hence received a lot of attention.

In this work, we prove tighter lower bounds on the number of linearly active S-boxes in CLEFIA-type generalized Feistel networks (GFNs) with diffusion switching mechanism (DSM). We show that every 6 rounds of such GFNs provide 50\% more linearly active S-boxes than proven previously. Moreover, we experimentally demonstrate that the new bound is tight for up to at least 12 rounds, whereas the previous one is not.

Thus, this paper delivers first provable evidence that diffusion switching mechanism actually provides an advantage by guaranteeing more active S-boxes in GFNs.
\end{abstract}

Keywords: block ciphers, generalized Feistel networks, CLEFIA, diffusion switching mechanism, substitution diffusion networks, linear cryptanalysis, efficiency

\section{Introduction}

\subsection{Motivation}

Generalized Feistel networks (GFNs) [18] have been popular with the designers of symmetric-key cryptographic primitives including block ciphers, stream ciphers and hash functions. They offer a simple way of domain extension given a function with good cryptographic properties. Probably the best understood structure of its round transform relies on substitution-diffusion functions (SD-functions) - a brick layer of local nonlinear permutations ( $S$-boxes) followed by a multiplication by a

Email addresses: qingju.wang@esat.kuleuven.be (Qingju Wang), andrey.bogdanov@esat.kuleuven.be (Andrey Bogdanov) diffusion matrix over a binary finite field (linear diffusion).

$\mathrm{GFN}_{4}$ are 4-line generalized Feistel networks. Type-I and type-II $\mathrm{GFN}_{4}$ are referred to as $\mathrm{GFN}_{4}-$ I and $\mathrm{GFN}_{4}$-II throughout this paper, structures are shown in Figure 1 and 2. The findings of [5] indicate that going from single SD-functions $[19,11]$ to double SD-functions improves the efficiency of $\mathrm{GFN}_{4}$ by up to $33 \%$ for $\mathrm{GFN}_{4}$-I and by up to $50 \%$ for $\mathrm{GFN}_{4}$-II, as measured by the proportion of differentially and linearly S-boxes in all S-boxes of the cipher. The work [5] proves that for $\mathrm{GFN}_{4}$ with double SD-functions every 14 rounds of $\mathrm{GFN}_{4}$-I and every 6 rounds of $\mathrm{GFN}_{4}$-II add $7 \mathcal{B}$ and $6 \mathcal{B}$ differentially and linearly active 


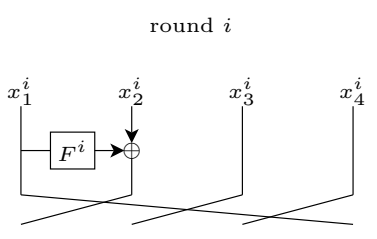

Figure 1: $\mathrm{GFN}_{4}$-I

S-boxes, respectively, where $\mathcal{B}$ is the branch number of the diffusion matrix $M$ (or its transpose) used in the round functions. Underlying SD-type functions can differ depending on:

- Number of distinct diffusion matrices: The standard approach is to use a single matrix in all rounds and functions (singleround diffusion), e.g. applied in Camellia [1]. The alternative approach proposed in [13] is to employ two and more distinct diffusion matrices in different rounds and functions (multiple-round diffusion, or diffusion switching mechanism, DSM), which prevents difference and linear mask cancelation at XORs, e.g. utilized in CLEFIA [14].

- Number of SD-layers in a function: SDtype functions usually consist of a single SDlayer (single SD-functions), as e.g. those in CLEFIA [14] and Camellia [1]. In some ciphers, however, SD-type functions have double SD-layers (double SD-functions), e.g. in E2 [8] and Piccolo [12].

CLEFIA is a popular recent block cipher designed by Sony Corporation, accepted as a lightweight encryption algorithm of the new ISO/IEC 29192-2 standard, and proposed as a CRYPTREC Japaneese e-Government recommendation cipher. The design of CLEFIA is a 4-line type-II GFN $\left(G F N_{4}-I I\right)$ with DSM and single SD-functions. $\mathrm{GFN}_{4}$-II belongs to the type of GFNs. We will be investigating in this paper $-d$-line type-II GFNs with DSM and single SD-functions, $\mathrm{GFN}_{d}$-II.

\subsection{Previous work on $G F N_{d}-I I$}

$\mathrm{GFN}_{d}$-II with other types of SD-type functions have been thoroughly studied in the literature, see Table 1.

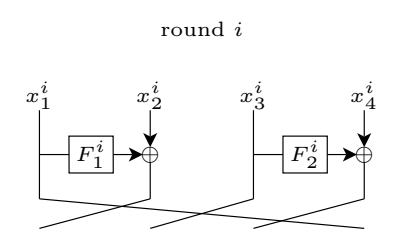

Figure 2: CLEFIA-type $\mathrm{GFN}_{4}$-II

Tight lower bounds on the number of both differentially and linearly active S-boxes for $\mathrm{GFN}_{4}$-II with single SD-functions and single-round diffusion are obtained in [11]: Every 6 rounds of $\mathrm{GFN}_{4^{-}}$ II are proven to provide at least $2 \mathcal{B}$ active $\mathrm{S}$-boxes, where $\mathcal{B}$ is the differential and linear branch number of the diffusion matrices used in the round functions.

Tight minimum numbers of differentially and linearly active S-boxes for $\mathrm{GFN}_{4}$-II with double SD-functions and single-round diffusion are proven in [5]. The findings of [5] indicate that going from single SD-functions [11] to double SD-functions improves the efficiency of $\mathrm{GFN}_{4}$-II by up to $50 \%$, as measured by the proportion of differentially and linearly S-boxes in all S-boxes of the cipher. The work [5] proves that every 6 rounds of $\mathrm{GFN}_{4}{ }^{-}$ II with double SD-functions add at least $6 \mathcal{B}$ active S-boxes for both differential and linear cryptanalysis.

Bounds on the number of differentially and linearly active S-boxes for $\mathrm{GFN}_{4}$-II with single SDfunctions and DSM were obtained in [15]. It is proven that every 6 rounds add at least $2 \mathcal{B}$ differentially and linearly active S-boxes. However, this bound is not tight, especially for the number of linearly active S-boxes. In fact, the bound proven in [15] for DSM yields a lower number of active S-boxes than for single-round diffusion. So there has been no proof so far that DSM has any advantage over single-round diffusion. This paper will greatly improve upon this.

\subsection{Contributions of this paper}

In this work, we prove that every 6 rounds of $\mathrm{GFN}_{d^{-}}$II with multiple-round diffusion (diffusion switching mechanism) and single SD-functions add at least $3 \mathcal{B}$ linearly active S-boxes, see Table 1 . This is exactly the construction behind the design of the lightweight block cipher CLEFIA, for 
Table 1: Upper bounds on the number of linearly active S-boxes and efficiency $E$ (Definition 1) for GFN ${ }_{d}$-II with SD-type functions.

\begin{tabular}{l|c|c|l|l|c|c} 
design & rounds & bound & function & diffusion & tightness & efficiency $E$ \\
\hline \hline GFN $_{4}$-II [11] & 6 & $2 \mathcal{B}+2$ & single SD & single-round & yes & $1 / 6$ \\
\hline GFN $_{4}$-II [5] & 6 & $6 \mathcal{B}$ & double SD & single-round & yes & $1 / 4^{*}$ \\
\hline GFN $_{d}$-II [15] & 6 & $2 \mathcal{B}$ & single SD & DSM & no & $1 / 6$ \\
\hline \hline GFN $_{d}$-II, here & 6 & $3 \mathcal{B}$ & single SD & DSM & yes & $1 / 4$
\end{tabular}

* Note that one has a doubled number of S-boxes in a round for double SD-functions

$\mathcal{B}$ : See Definitions 3 and 4

the case of $d=4$. $\mathcal{B}$ is the branch number of the single- and multiple-round diffusion matrices (their transposed inverses) used in the round functions. We experimentally demonstrate that the new bound is tight for up to at least 12 rounds, whereas the previous one is not.

The relevance of this bound is three-fold:

- This result indicates that the efficiency of CLEFIA-type GFNs is 50\% higher than previously proven, in terms of the proportion of linearly active S-boxes in all S-boxes. This efficiency metric $E$ is a valid efficiency metric introduced in [16] and used in [2, 3, 4, 5]. Its definition can be found in Definition 1 .

- Moreover, the new result suggests that the efficiency of $\mathrm{GFN}_{d}$ with SD-type functions is equally improved both by moving from single to double SD-functions [5] and by going from single-round diffusion to DSM over multiple rounds - the central contribution of this paper.

- The new bounds is also the first provable evidence that GFNs with DSM can actually provide more active S-boxes than GFNs with single-round diffusion. Previously [14, 15], for GFNs, this advantage has been only demonstrated numerically for some concrete CLEFIA-like examples.

\section{Preliminaries}

\section{1. $G F N_{4}$ with $S D$-functions}

Type-I and type-II GFNs are block ciphers with the state equally divided into an even num-

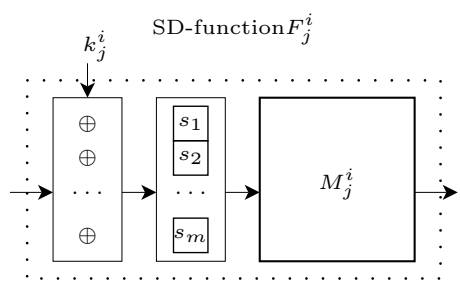

Figure 3: SD-function

ber $d \geq 4$ wide lines. They are referred to as $\mathrm{GFN}_{d^{-}} \mathrm{I}$ and $\mathrm{GFN}_{d^{-}} \mathrm{II}$ in this paper. The structurs of $\mathrm{GFN}_{d^{-}} \mathrm{I}$ and $\mathrm{GFN}_{d^{-}}$II when $d=4$ are as shown in Figure 1 and 2. In one round of both $\mathrm{GFN}_{4}$-I and $\mathrm{GFN}_{4}$-II, let the input $x^{i}$ of round $i$ be $x^{i}=$ $\left(x_{1}^{i}, x_{2}^{i}, x_{3}^{i}, x_{4}^{i}\right)$. Then the output of $\mathrm{GFN}_{4}$-I and and for $\mathrm{GFN}_{4}$-II will be $\left(x_{2}^{i} \oplus F^{i}\left(x_{1}^{i}\right), x_{3}^{i}, x_{4}^{i}, x_{1}^{i}\right)$ and $\left(x_{2}^{i} \oplus F_{1}^{i}\left(x_{1}^{i}\right), x_{3}^{i}, x_{4}^{i} \oplus F_{2}^{i}\left(x_{3}^{i}\right), x_{1}^{i}\right)$ respectively, for some keyed nonlinear functions $F^{i}, F_{1}^{i}$ and $F_{2}^{i}$. The $j$-th F-functions $F_{j}^{i}$ of round $i$ often exhibit the Substitution-Diffusion (SD) structure

(For type-I, there is only one F-function in each round). Here, the subkey addition followed by a layer of $m$ S-boxes, $s_{i}, i=1, \ldots, m$, and an $m \times m$ linear diffusion mapping $M_{j}^{i}$ over a binary finite field. Such F-functions are called SD-functions. The structure of SD-functions is depicted in Figure 3 .

\subsection{Diffusion Switching Mechanism}

Diffusion Switching Mechanism (DSM) is a design approach for Feistel networks proposed by Shirai and Shibutani [13] and used in CLEFIA [14]. In this technique, two or more distinct diffusion matrices in the round function are switched among multiple rounds in a predefined order to prevent 
the difference (linear mask) cancellation which occurs in the differential (linear) trails due to the fact that only a single matrix is employed for linear diffusion. Therefore, it provides a larger number of active S-boxes than the single-round diffusion and is adopted to enhance the efficiency of Feistel ciphers against differential and linear cryptanalysis. A linearly active S-box is defined as an S-box given the non-zero input linear mask.

\subsection{Efficiency Metrics}

Here a definition of two popular efficiency metrics for ciphers with SD-functions is given as introduced by Shirai and Preneel in [16]:

Definition 1. (Efficiency metrics [16]) The efficiency metric $E_{m, r}$ for a $G F N_{d}-I I$ cipher over $r$ rounds is defined as

$$
E_{m, r}=\frac{A_{m, r}}{S_{m, r}}
$$

where $A_{m, r}$ is the number of active $S$-boxes over $r$ rounds and $S_{m, r}$ is the total number of $S$-boxes over $r$ rounds. The efficiency metric $E$ is defined as

$$
E=\lim _{m, r \rightarrow \infty} E_{m, r},
$$

which simplifies comparisons.

\section{Minimum number of active S-boxes}

In this section, we prove a lower bound on the number of linearly active S-boxes for six rounds $\mathrm{GFN}_{d^{-}}$II $(d \geq 4)$ with DSM, the untwisted form of which is illustrated in Figure 4. When the DSM design strategy is applied to $\mathrm{GFN}_{d}$-II, from Figure 4 we can see that the relation between two matrices $M_{j}^{i}$ in $F_{j}^{i}$ and $M_{j-1}^{i+2}$ in $F_{j-1}^{i+2}$ for all possible $i$ and $j$ should be considered.

\subsection{Branch numbers}

First we recall the notions of branch numbers we will be using throughout the section:

Definition 2. (Bundle weight [6]) Let $x \in\{0,1\}^{p n}$ be represented as $x=\left(x_{0}, x_{1}, \cdots, x_{p-1}\right)$, where $x_{i} \in\{0,1\}^{n}$, then the bundle weight $w_{n}(x)$ is defined as

$$
w_{n}(x)=\#\left\{i \mid 0 \leq i \leq p-1, x_{i} \neq 0\right\} .
$$

Definition 3. (Branch number [6]) Let permutation $P:\{0,1\}^{p n} \rightarrow\{0,1\}^{q n}$. The branch number of $P$ is defined as

$$
\mathcal{B}_{n}(P)=\min _{a \neq 0}\left\{w_{n}(a)+w_{n}(P(a))\right\} .
$$

Similarly to Definition 3, one can define a linear branch number for two matrices:

Definition 4. (Linear branch number of multiple matrices [16]) The linear branch number of two matrices is defined as

$$
\mathcal{B}^{L}=\min \left\{\mathcal{B}_{n}\left[\left.{ }^{t}\left(M_{j}^{i}\right)^{-1}\right|^{t}\left(M_{j-1}^{i+2}\right)^{-1}\right]\right\} .
$$

where ${ }^{t} M$ denotes the transpose of matrix $M,[M \mid N]$ denotes an $m \times 2 m$ matrix obtained by concatenating two $m \times m$ matrices $M$ and $N$.

\subsection{Two basic properties}

With respect to the $j$-th F-function $F_{j}^{i}$ in the $i$ th round, we denote $\Gamma x_{j}^{i}$ and $\Gamma y_{j}^{i}$ as the input and output linear masks, $L_{j}^{i}$ as the number of linearly active S-boxes in $F_{j}^{i}$. Also $L^{i}$ denotes the number of linearly active S-boxes in the $i$-th round and, thus, $L^{i}=\sum_{j=0}^{\frac{d}{2}-1} L_{j}^{i}$. When DSM design strategy is employed in $\mathrm{GFN}_{d}$-II, the following properties hold:

Property 1. Any two consecutive rounds of GFNs have at least one linear active F-function if a nonzero linear mask input is given.

If the input linear mask in the first round is non-zero, the input lines added to the output of Ffuntion in the first round will become the input of the F-function in the next round, it means that the input of both F-funtions in the consecutive two rounds can not be zero at the same time.

The following property is derived in [15]:

Property 2. For any $i$ and $j$, the set of $L_{j}^{i}, L_{j}^{i+1}$ and $L_{j-1}^{i+2}$ satisfies one of the two cases:

- $L_{j}^{i}=L_{j}^{i+1}=L_{j-1}^{i+2}=0$;

- $L_{j}^{i}+L_{j}^{i+1}+L_{j-1}^{i+2} \geq \mathcal{B}^{L}$, where two of the terms are always non-zero. 


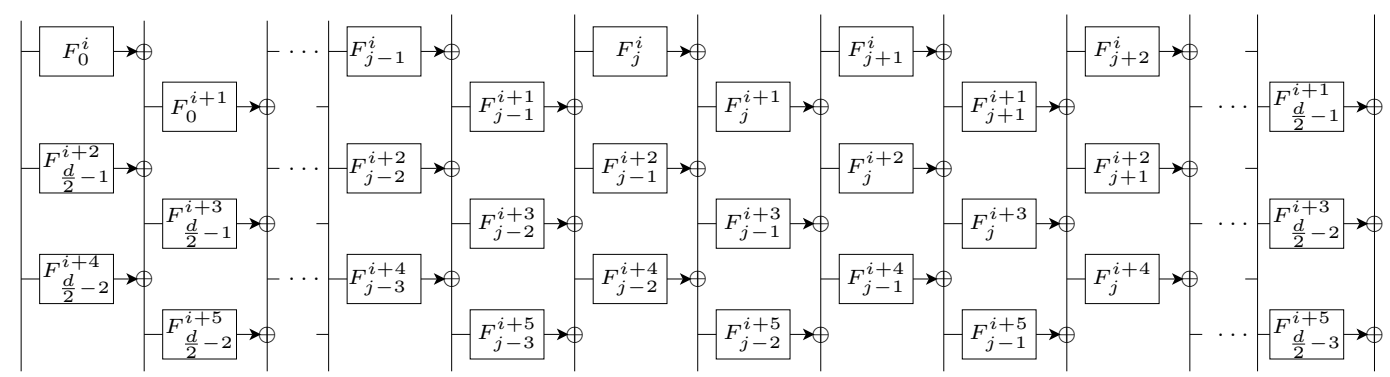

Figure 4: 6-round $\mathrm{GFN}_{d}$-II with DSM

Three round linear mask relation $\Gamma x_{j}^{i+1}=\Gamma y_{j}^{i} \oplus$ $\Gamma y_{j-1}^{i+2}$ can be represented in matrix form as $\Gamma x_{j}^{i+1}=$ ${ }^{t}\left(M_{j}^{i}\right)^{-1} \Gamma x_{j}^{i} \oplus^{t}\left(M_{j-1}^{i+2}\right)^{-1} \Gamma x_{j-1}^{i+2}$. If any two of the above terms are zero, it is obviously all the three are zero. Using the the notion of the branch number $L_{j}^{i}$, it implies the first case in Property 2; otherwise if any of the three terms is non-zero, we can see at least two terms will be non-zero. In addition, from

$$
\Gamma x_{j}^{i+1}=\left[\left.{ }^{t}\left(M_{j}^{i}\right)^{-1}\right|^{t}\left(M_{j-1}^{i+2}\right)^{-1}\right]\left(\begin{array}{c}
\Gamma x_{j}^{i} \\
\Gamma x_{j-1}^{i+2}
\end{array}\right)
$$

and since $\left.\left.{ }^{t}\left(M_{j}^{i}\right)^{-1}\right|^{t}\left(M_{j-1}^{i+2}\right)^{-1}\right]$ has a branch number at least $\mathcal{B}^{L}$, we obtain

$$
w_{n}\left(\Gamma x_{j}^{i}\right)+w_{n}\left(\Gamma x_{j}^{i+1}\right)+w_{n}\left(\Gamma x_{j-1}^{i+2}\right) \geq \mathcal{B}^{L},
$$

and, thus, the second case of Property 2 is shown.

\subsection{Main result}

Based on the above two properties, now we can derive the main result of the paper:

Theorem 1. Let $d \geq 4$. Any consecutive six rounds of $G F N_{d}-I I$ with DSM guarantee at least $3 \mathcal{B}^{L}$ linear active $S$-boxes.

Proof. We consider six consecutive rounds starting in the $i$-th round. Property 1 implies that at least one $\mathrm{F}$-function has a non-zero linear mask in the 3 rd or 4 th round, i.e. the $(i+2)$-th or the $(i+3)$-th round. We consider them separately as Case 1 and Case 2.

Case 1. A non-zero linear mask exists in the 3rd round, i.e. $L_{j}^{i+2} \neq 0$.
Based on condition $L_{j}^{i+2} \neq 0$ and Property 2, we get

$$
L_{j+1}^{i}+L_{j+1}^{i+1}+L_{j}^{i+2} \geq \mathcal{B}^{L}
$$

and $L_{j}^{i+1}+L_{j-1}^{i+3} \geq 1$. Now we consider two subcases $L_{j}^{i+1} \neq 0$ and $L_{j-1}^{i+3} \neq 0$ as follows:

Case 1-1. If $L_{j}^{i+1} \neq 0$, Property 2 implies that

$$
L_{j}^{i}+L_{j}^{i+1}+L_{j-1}^{i+2} \geq \mathcal{B}^{L} .
$$

Similarly, from condition $L_{j}^{i+2} \neq 0$ and Property $2, L_{j-1}^{i+4}+L_{j}^{i+3} \geq 1$, i.e. at least one of the inequalities can be obtained from $L_{j-1}^{i+4} \neq 0$ and $L_{j}^{i+3} \neq 0$ respectively:

$$
\begin{gathered}
L_{j-1}^{i+3}+L_{j-1}^{i+4}+L_{j-2}^{i+5} \geq \mathcal{B}^{L} \\
L_{j}^{i+3}+L_{j}^{i+4}+L_{j-1}^{i+5} \geq \mathcal{B}^{L}
\end{gathered}
$$

If $d=4, F_{j}^{i+4}$ will be $F_{j-2}^{i+4}$. Thus (4) will be $L_{j}^{i+3}+L_{j-2}^{i+4}+L_{j-1}^{i+5} \geq \mathcal{B}^{L}$. Therefore from the above inequalities (1), (2) and (3) or (1), (2) and (4) (even when $d=4$, there is no overlapped term in the three inequalities), we get that in this case the minimum number of linearly active S-boxes is $\sum_{k=i}^{i+5} \sum_{j=0}^{\frac{d}{2}-1} L_{j}^{k} \geq$ $3 \mathcal{B}^{L}$.

Case 1-2. If $L_{j-1}^{i+3} \neq 0$, Property 2 guarantees (3) again. From condition $L_{j-1}^{i+3} \neq$ 0 and Property $2, L_{j-1}^{i+2}+L_{j-2}^{i+4} \geq 1$ can be obtained. Therefore at least one of the two inequalities (2) and (5) with

$$
L_{j-2}^{i+3}+L_{j-2}^{i+4}+L_{j-3}^{i+5} \geq \mathcal{B}^{L}
$$


can be derived from Property 2 , since of $L_{j-1}^{i+2} \neq 0$ and $L_{j-2}^{i+4} \neq 0$, respectively. If $d=4, F_{j-2}^{i+3}$ and $F_{j-3}^{i+5}$ will become $F_{j}^{i+3}$ and $F_{j-1}^{i+5}$ respectively, and (5) will be $L_{j}^{i+3}+L_{j-2}^{i+4}+L_{j-1}^{i+5} \geq \mathcal{B}^{L}$. Therefore based on the above inequalities (1), (3) and (2) or inequalities (1), (3) and (5) (when $d=4$, there are still no overlapped terms), we can conclude that in this case $\sum_{k=i}^{i+5} \sum_{j=0}^{\frac{d}{2}-1} L_{j}^{k} \geq 3 \mathcal{B}^{L}$.

Case 2. Any non-zero linear mask exists in the 4th round, i.e. $L_{j-1}^{i+3} \neq 0$.

Since $L_{j-1}^{i+3} \neq 0$, from Property 2, (3) and $L_{j-1}^{i+2}+L_{j-2}^{i+4} \geq 1$ can be derived. Again, similarly to Case 1, two subcases will be considered as follows, $L_{j-1}^{i+2} \neq 0$ and $L_{j-2}^{i+4} \neq 0$ :

Case 2-1. If $L_{j-1}^{i+2} \neq 0$, Property $2 \mathrm{im-}$ plies (2). Similarly based on condition $L_{j}^{i+2} \neq 0$ and Property 2, at least one of the two inequalities (5) and (6) with

$$
L_{j-1}^{i}+L_{j-1}^{i+1}+L_{j-2}^{i+2} \geq \mathcal{B}^{L}
$$

can be obtained. When $d=4$, (3) becomes $L_{j-1}^{i+3}+L_{j-3}^{i+4}+L_{j-2}^{i+5} \geq \mathcal{B}^{L}$. Thus the above inequalities (3), (2) and (5) or inequalities (3), (2) and (6), guarantee that the minimum number of active S-boxes is $\sum_{k=i}^{i+5} \sum_{j=0}^{\frac{d}{2}-1} L_{j}^{k} \geq 3 \mathcal{B}^{L}$.

Case 2-2. If $L_{j-2}^{i+4} \neq 0$, Property 2 means that (5) holds. Moreover, from condition $L_{j-1}^{i+3} \neq 0$ and Property $2, L_{j}^{i+1}+$ $L_{j}^{i+2} \geq 1$ can be obtained. Therefore at least one of the two equalities (2) and (1) can be derived from Property 2 since $L_{j}^{i+1} \neq 0$ and $L_{j}^{i+2} \neq 0$, respectively. When considering $d=4$, (5) will be turned into $L_{j}^{i+3}+L_{j-2}^{i+4}+L_{j-1}^{i+5} \geq$ $\mathcal{B}^{L}$. Therefore inequalities (3), (5) and (1) or inequalities (3), (5) and (2), yield $\sum_{k=i}^{i+5} \sum_{j=0}^{\frac{d}{2}-1} L_{j}^{k} \geq 3 \mathcal{B}^{L}$.

Combining all the above cases, we can conclude that at least $3 \mathcal{B}^{L}$ linear active $\mathrm{S}$-boxes are guaranteed in any six consecutive rounds of $\mathrm{GFN}_{d}$-II with DSM $(d \geq 4)$ which yields the claim of the theorem.
Therefore, we have proven that every six rounds $\mathrm{GFN}_{d}$-II with DSM $(d \geq 4)$ provide a tight upper bound of $3 \mathcal{B}$ on the number of linearly active S-boxes. When $d=4$, it is CLEFIA-type GFNs and, thus, we showed that any six rounds of CLEFIA-type GFNs yield by $50 \%$ more linearly active S-boxes than previously proven in [15], see also Table 1.

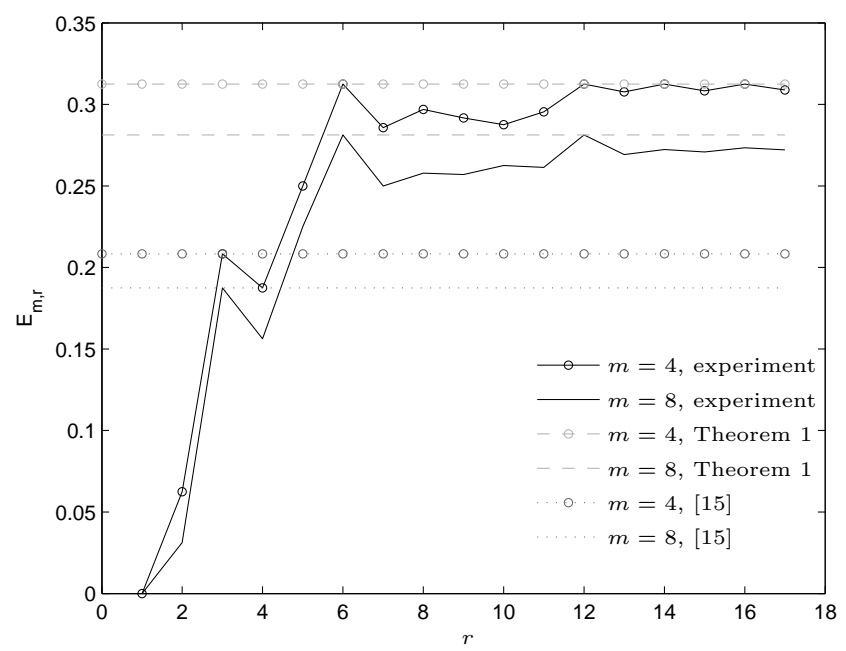

Figure 5: Experimental efficiency $E_{m, r}$ and bounds, $m \in\{4,8\}$, for $\mathrm{GFN}_{4}$-II. The new bound is tight e.g. for $r=6$ and $r=12$

\section{Tightness, experiments and conclusions}

We investigate two examples of $\mathrm{GFN}_{4}$-II with DSM and MDS diffusion matrices [9, 6] to show tightness of the bounds of Theorem 1: one with $m=4$ and one with $m=8$. Note that $\mathcal{B}^{L}=$ $m+1$ for MDS matrices. For these constructions, we experimentally derive the actual numbers $A_{m, r}$ of linearly active S-boxes over several rounds $r$. Then, using metric $E_{m, r}$ of Definition 1 , we numerically compute the efficiency of this construction over $r$ rounds. These results are given in Figure 5, together with the bounds of Theorem $1,3 \mathcal{B}^{L}=3(m+1)$, as well as those of [15], $2 \mathcal{B}^{L}=2(m+1)$.

To obtain the experimental results of Figure 5 , we adopt the mixed-integer linear programming 
(MILP) technique proposed in [10] to find the security bounds for the initialization phase of the Hitachi-designed stream cipher Enocoro-128v2 [17] against both differential and linear cryptanalysis. The optimizer CPLEX [7] is used in our implementation of the technique.

We observe that our bound appears tight for at least both $r=6$ and $r=12$ rounds, whereas the bound of [15] is not. Moreover, we see that considering multiples of 6 rounds for the bound on the number of linearly active S-boxes is the most informative choice since it is those numbers of rounds that provide most efficiency.

We conclude with the observation that the bound of Theorem 1 delivers first provable evidence that employing the diffusion switching mechanism in GFN designs (and especially, in CLEFIAtype GFNs) actually provides an advantage by guaranteeing more active S-boxes.

Acknowledgements. Andrey Bogdanov is postdoctoral fellow of the Fund for Scientific Research - Flanders (FWO). This work was supported in part by the National Natural Science Foundation of China (No. 61073150), by the IAP Programme P6/26 BCRYPT of the Belgian State, by the European Commission under contract number ICT2007-216676 ECRYPT NoE phase II, by KU LeuvenBOF (OT/08/027), and by the Research Council KU Leuven (GOA TENSE).

\section{References}

[1] Aoki, K., Ichikawa, T., Kanda, M., Matsui, M., Moriai, S., Nakajima, J., Tokita, T.: Camellia: A 128-Bit Block Cipher Suitable for Multiple Platforms - Design and Analysis. In: D.R. Stinson and S. Tavares, editors, SAC'00, vol. 2012 of LNCS, pp. 3956, Springer-Verlag (2001)

[2] Bogdanov, A.: On the Differential and Linear Efficiency of Balanced Feistel Networks. Information Processing Letters 110(20), pp. 861-866, Elsevier (2010)

[3] Bogdanov, A.: On Unbalanced Feistel Networks with Contracting MDS Diffusion. Designs, Codes and Cryptography 59(1-3), pp. 35-58. Springer-Verlag (2011)

[4] Bogdanov, A., Shibutani, K.: Double SP-Functions: Enhanced Generalized Feistel Networks. In: U. Parampalli and P. Hawkes (eds.), ACISP 2011, LNCS, vol. 6812, pp. 106-119, Springer-Verlag (2011)
[5] Bogdanov, A., Shibutani, K.: Generalized Feistel Networks Revisited. WCC'11, Workshop on Coding and Cryptography (2011). Submitted to Designs, Codes and Cryptography (2011)

[6] Daemen, J., Rijmen, V.: The Design of Rijndael: AES - The Advanced Encryption Standard. Information Security and Cryptography, Springer-Verlag (2002)

[7] IBM: IBM ILOG CPLEX Optimizer.

[8] Kanda, M., Moriai, S., Aoki, K., Ueda, H., Takashima, Y., Ohta, K., Matsumoto, T.: E2 - A New 128-Bit Block Cipher. IEICE Trans. Fundamentals, vol. E83-A(1), January 2000.

[9] Lidl, R., Niederreiter, H.: Finite Fields. Encyclopedia of Mathematics and Its Applications 20. Cambridge University Press (1997)

[10] Mouha, N., Wang, Q., Gu, D., Preneel, B.: Differential and Linear Cryptanalysis using MixedInteger Linear Programming. In: Inscrypt'11. LNCS, Springer-Verlag (2011)

[11] Shibutani, K.: On the Diffusion of Generalized Feistel Structures Regarding Differential and Linear Cryptanalysis. In: SAC'10. LNCS, Springer-Verlag (2010)

[12] Shibutani, K. Isobe, T., Hiwatari, H., Mitsuda, A., Akishita, T., Shirai, T.: Piccolo: An UltraLightweight Blockcipher. In: B. Preneel and T. Takagi, editors, CHES'11, vol. 6917 of LNCS, SpringerVerlag (2011)

[13] Shirai, T., Shibutani, K.: Improving immunity of feistel ciphers against differential cryptanalysis by using multiple mds matrices. In: FSE'04, LNCS, vol. 3017, pp. 260-278. Springer-Verlag (2004)

[14] Shirai, T., Shibutani, K., Akishita, T., Moriai, S., Iwata, T.: The 128-Bit Blockcipher CLEFIA (Extended Abstract). In: FSE'07. LNCS, vol. 4593, pp. 181-195. Springer-Verlag (2007)

[15] Shirai, T., Araki, K.: On Generalized Feistel Structures Using the Diffusion Switching Mechanism. IEICE Transactions 91-A(8), pp. 2120-2129 (2008)

[16] Shirai, T., Preneel, B.: On Feistel Ciphers Using Optimal Diffusion Mappings Across Multiple Rounds. In: ASIACRYPT'04. LNCS, vol. 3329, pp. 1-15. Springer-Verlag (2004)

[17] Watanabe, D., Okamoto, K., Kaneko, T.: A Hardware-Oriented Light Weight Pseudo-Random Number Generator Enocoro-128v2. In: The Symposium on Cryptography and Information Security. pp. 3D1-3 (2010)

[18] Zheng, Y., Matsumoto, T., Imai, H.: On the Construction of Block Ciphers Provably Secure and Not Relying on Any Unproved Hypotheses. In: CRYPTO'89. LNCS, vol. 435, pp. 461-480. SpringerVerlag (1989)

[19] Wu, W., Zhang, W., Lin, D.: Security on Generalized Feistel Scheme with SP Round Function. I. J. Network Security 3(3), 215-224 (2006) 